\section{A FLEXIBLE, FUNCTIONAL AND STYLISH ADDITION TO YOUR SURGERY}

Henry Schein Dental is delighted to introduce the new INTEGO - the very latest in high quality treatment centres from Sirona.

Beautifully built, compact and available in a range of vibrant colours to suit all surgery configurations and styles, the INTEGO effortlessly combines high quality, hard-wearing materials with an award winning design.

INTEGO's innovative features focus on ergonomic operating procedures and total patient comfort. A user-friendly and intuitive EasyPad interface means managing your workflow has never been easier. It also features a flexible compact water unit for optimal use of space and controlled water quality, giving assurance of hygiene and infection control.

You may think a treatment centre of this quality would come with an extravagant price tag, but the INTEGO is very affordable and provides a flexible, functional and stylish addition to any surgery.

The first 25 Integos purchased (terms and conditions apply) will receive six FREE Sirona turbines or contraangles - call 08700102041 to find out more.

www.henryschein.co.uk

\title{
PERFECTLY MIXED, BUBBLE-FREE IMPRESSIONS
}

MixStar-eMotion is DMG UK's latest generation fully automatic mixing machine. Designed for use with their market leading MixStar impression materials - Honigum-MixStar, Silagum-MixStar and StatusBlue - it means automatic mixing just gets better and better.

The MixStar-eMotion possesses three different, electronically controlled speed settings that ensure consistent results even with more viscous materials. The top speed enables dispensing at twice the speed of its predecessor. Setting and working time in the mouth can be adjusted with two timers and saved in programmes. The mixing process can be started with a simple press of a button or by using an optional footswitch, which can be plugged in for hands-free operation. With the aid of a serial interface, software can be updated anytime via the Internet.

The MixStar-eMotion mixing unit is a fully automatic mixing machine designed to deliver perfectly mixed, homogenous, bubble-free impression material directly into the impression tray or syringe. Extremely user-friendly, it is easy to operate at the touch of a button, enabling both of the operator's hands to remain free to hold the tray or syringe during filling. It offers user-friendly mixing and dispensing speeds plus quick and easy cartridge and mixing tip exchange when required.

Used in combination with DMG's MixStar range of impression materials it helps save valuable surgery time, waste and mess.

For further information contact your local dental dealer or DMG Dental Products (UK) Ltd on 01656789401 or visit www.dmgdental.com.

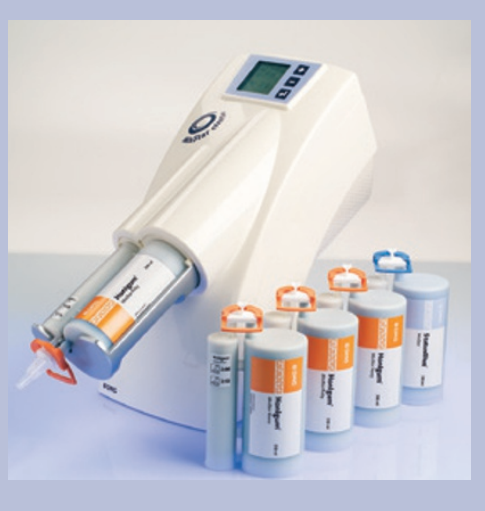

\section{MAKE USE OF SPACE,} COLOUR AND LIGHT

Being scared of the dentist is often the reason given for patients not attending regular appointments. Make your practice a welcoming environment for everyone by giving it a redesign with the help of Tavom UK.

Dental phobia often starts

from a childhood association with unpleasant sights, sounds and smells of a surgery. But modern practices can be a much friendlier environment. By making use of space, colour and light, nervous patients can be soothed the minute they enter and feel confident that they are in safe and professional hands.

Tavom UK is the expert in practice redesign. With years of experience manufacturing bespoke cabinetry for the medical and dental industries, its team will help to design the perfect modern workspace. With beautiful cabinets and benches available in a range of colours and finishes, a practice refreshed by Tavom UK will keep any memories of gloomy and intimidating surgeries firmly in the past.

Tavom UK will projectmanage your redesign from beginning to end, and provide ongoing customer service that is second-to-none. Call today to find out more on 08707521121 or visit www.tavomuk.com.

\section{ALLEVIATE SYMPTOMS OF PROBLEM SNORING}

Patients can now immediately receive an affordable, pro-quality oral appliance $(\mathrm{OA})$ to alleviate symptoms of problem snoring and obstructive sleep apnoea (OSA). BluePro from BlueSom can be fitted quickly by trained dentists without the high cost and delay associated with lab-made appliances.

Snoring is a common symptom of OSA which if left untreated can disrupt sleep and lead to serious medical problems.
The first-line treatment for severe OSA is continuous positive airway pressure (CPAP) which is often limited by poor patient adherence. Good quality custom-made OAs fitted by dentists can be an effective alternative treatment for non-compliant CPAP patients. OAs are also used as a first-line treatment for non-apnoeic snoring and mild to moderate OSA. Unfortunately they are unaffordable for many patients and typically involve a delay of several weeks between diagnosis and treatment.

BluePro was designed by experts in oral appliance technology. It can be fitted immediately by heating in boiling water and taking a direct impression of the teeth. The customised mouthpieces are joined by a mechanism allowing accurate adjustments to advance the lower jaw and keep the patient's airway open comfortably during sleep. BluePro effectively reduces symptoms of OSA and has a predicted usable life of one year. http://www. bluepro.pro/ en.

IUP 